\title{
A Case of Angioleiomyoma with Epithelioid Granuloma
}

\author{
Masazumi Onishi Kazuhiro Takahashi Daisuke Watabe \\ Hanae Onodera Toshihide Akasaka \\ Department of Dermatology, Iwate Medical University, Morioka, Japan
}

\section{Key Words}

Angioleiomyoma $\cdot$ Sarcoidosis $\cdot$ Sarcoid reaction $\cdot$ Epithelioid granuloma

\begin{abstract}
We describe a 61-year-old Japanese woman who had been aware of a lesion on her left thigh for 10 years. Pathological examination demonstrated a well-circumscribed encapsulated nodule at the dermal-subcutaneous boundary, composed of eosinophilic spindle cell bundles, connective tissue, and numerous small vessels.

Immunohistochemically, these eosinophilic cells were positive for a-smooth muscle actin. The granulomatous areas in the tumor were composed focally of epithelioid cells and lymphocytes. The epithelioid cells were negative for a-smooth muscle actin. We diagnosed this case as an angioleiomyoma with epithelioid granuloma. Malignant tumors with granulomatous change have sometimes been reported in the literature, but benign tumors with epithelioid granuloma, such as the present one, are rare. We thought that epithelioid cell granuloma might transform to angioleiomyoma through the action of IL-1 released from vascular smooth muscle cells.
\end{abstract}

\section{Introduction}

Sarcoidosis is characterized by the presence of epithelioid granuloma without necrosis involving systemic organs. Sarcoid reactions composed of epithelioid cells and giant cells have been considered to affect only a single organ or regional lymph nodes against a background of underlying disease. Although a sarcoid reaction may often occur at the primary site of a malignant tumor, sarcoid reactions associated with benign tumors are very rare.

Here we describe a case of angioleiomyoma with epithelioid granuloma, and discuss the difference between sarcoidosis and a sarcoid reaction with reference to previous reports. 


\section{Case Report}

A 61-year-old Japanese woman presented with a painful, skin-colored, elastic-hard tumor $10 \mathrm{~mm}$ in diameter that had been present on her left thigh for 10 years (ig. 1 ). She also had impaired visual acuity due to cataract and uveitis, which was clinically suspected to be attributable to sarcoidosis. Otherwise, her medical history was unremarkable. No similar lesions were evident on other parts of the body. The results of laboratory tests were mostly within the normal range, including angiotensin-converting enzyme and lysozyme. Though the patient had been inoculated with BCG, a tuberculin skin test gave a negative result. A chest X-ray film demonstrated bilateral hilar lymphadenopathy. Echocardiography and 24-hour electrocardiographic Holter monitoring also demonstrated no abnormality. Subcutaneoustype sarcoidosis was suspected, and therefore we performed excisional biopsy of the tumor on the thigh.

Histopathologically, the nodule was well circumscribed and encapsulated at the dermalsubcutaneous boundary (fig. 2). The tumor consisted mainly of eosinophilic spindle cell bundles, connective tissue, and numerous small vessels (ig. 3il). Immunohistochemistry demonstrated that these eosinophilic cells were positive for $\alpha$-smooth muscle actin. Focally, areas of granulomatous reactivity composed of epithelioid cells and lymphocytes were evident within the tumor. The epithelioid cells were negative for $\alpha$-smooth muscle actin (fig. $3 b$ ). On the basis of the clinical presentation, and the histopathological and immunohistochemical findings, a diagnosis of angioleiomyoma with epithelioid granuloma was made.

\section{Discussion}

A sarcoid-like reaction (SLR) has been found occasionally in association with malignant tumors, such as Hodgkin's and non-Hodgkin's lymphomas, and carcinomas at different sites, and can occur in the context of the tumor itself, in metastatic lesions, or in lymph nodes draining the neoplasm. This reaction is a foreign-body inflammatory response to necrotic materials or an immunological reaction, the granulomatous response within draining lymph nodes representing a response to soluble tumor-related antigens or a stromal reaction representing a $\mathrm{T}$ cell-mediated immunological response to cell surface antigens [1]. However, only a few benign tumors with SLR similar to that in the present case of angioleiomyoma have been reported.

Two causes can be suggested for the pathogenesis of the granulomatous change in the present case: either a sarcoid reaction or generalized sarcoidosis. Our patient had associated uveitis and bilateral hilar lymphadenopathy, and the tuberculin test gave a negative result. Therefore it was suspected that the granuloma in the thigh tumor had arisen as part of a series of changes related to generalized sarcoidosis. To our knowledge, only three cases of benign tumors accompanied by epithelioid granuloma have been reported. Generalized sarcoidosis was present in all three cases (table 1) $[2,3]$.

Sarcoidosis is a chronic granulomatous disease of unknown etiology characterized by the development of sarcoid granulomas in several organs. Histopathologically, these granulomas are composed of accumulations of tightly packed macrophages and macrophage-derived giant cells. It has also been demonstrated that differentiation of cells induced by various cytokines can lead to the formation of sarcoid granuloma [4]. Prior et al. [4] cultured bronchoalveolar and peripheral blood mononuclear cells in serum-free medium, and analyzed the supernatants for the concentrations of interleukin-1 beta (IL$1 \beta)$, IL-2, IL-6, tumor necrosis factor-alpha (TNF- $\alpha$ ), granulocyte-macrophage colonystimulating factor (GM-CSF) and interferon-gamma (IFN- $\gamma$ ). Peripheral blood mononuclear cells from sarcoid patients were shown to release higher amounts of IL-1 $\beta$, TNF- $\alpha$, IL-6 and GM-CSF than those from normal controls [4]. Sarcoid inflammation 
was found to be associated with an increased and concerted release of monocyte/macrophage-derived cytokines, not only in the lung, but also in the peripheral blood [4]. Although we are unable to draw a definite conclusion with regard to the present case, these cytokines likely play an important role in the etiology of sarcoidosis.

On the other hand, leiomyomas are common dermal tumors arising from smooth muscle cells, and can develop in the erector pili muscle, vascular smooth muscle, scrotum, vulva, or mammilla [5]. Angioleiomyoma is a neoplasm of exclusively vascular smooth muscle (VSM) origin. VSM can release monocyte/macrophage-derived cytokines such as IL-1 and TNF- $\alpha[6,7]$. Accordingly, we considered that the VSM constituting angioleiomyoma might have produced immunostimulatory cytokines, which induced the formation of epithelioid cell granuloma. We conclude that epithelioid cell granuloma may transform to angioleiomyoma under the influence of IL-1, TNF- $\alpha$ and IL-6 released from VSM cells. In order to test this hypothesis, further investigation will be needed.

\section{Funding Sources}

None.

\section{Disclosure Statement}

The authors have no conflicts of interest.

Table 1, Benign tumors exhibiting epithelioid cell granuloma

\begin{tabular}{llllll}
\hline Case [reference] & Age & Sex & Basal disease & Site & $\begin{array}{l}\text { Generalized } \\
\text { sarcoidosis }\end{array}$ \\
\hline $1[2]$ & 54 & F & Pheochromocytoma & Abdominal cavity & $(+)$ \\
$2[3]$ & 43 & F & Leiomyoma & Uterus & $(+)$ \\
3 (present case) & 61 & F & Angioleiomyoma & Skin & $(+)$ \\
\hline
\end{tabular}




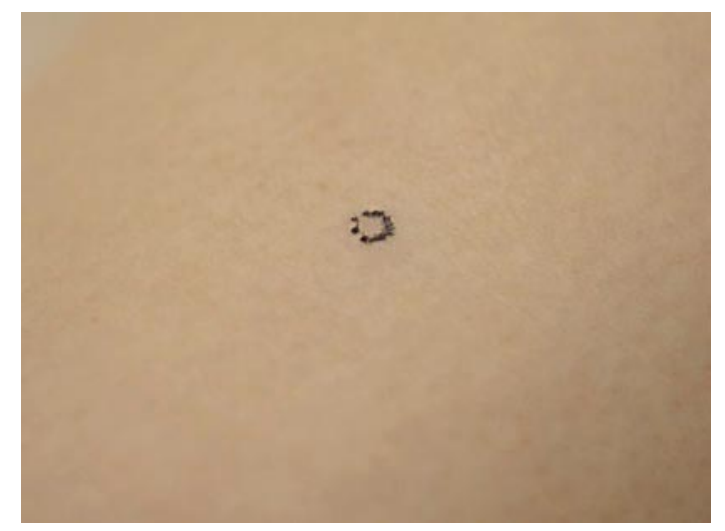

Fig. 1. Skin-colored, solitary, and elastic-hard subcutaneous nodule on the left thigh.

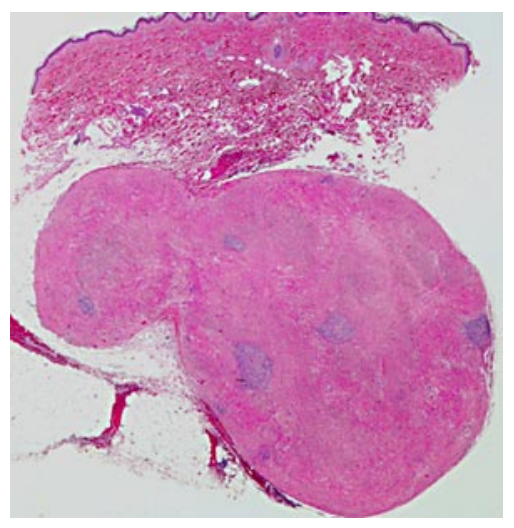

Fig. 2. Well-circumscribed tumor with inflammatory cell infiltration.
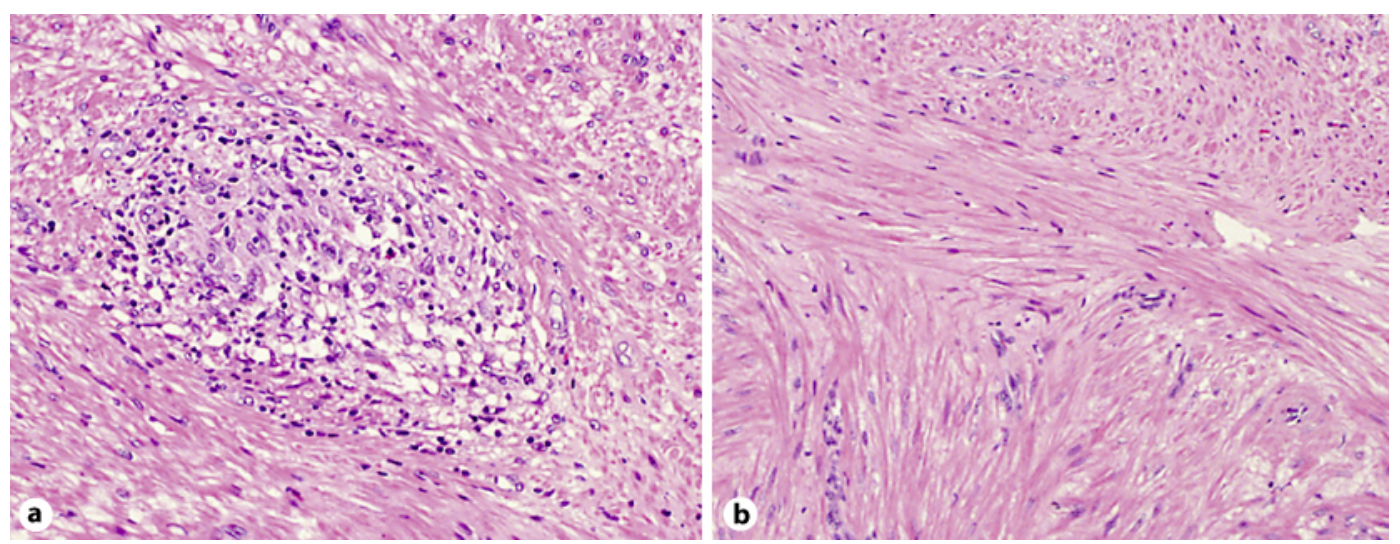

Fig. 3. a The tumor appears to be composed of eosinophilic spindle-shaped fasciculi, vascular spaces, and connective tissue. $\mathbf{b}$ Collections of epithelioid cells with lymphocytes are present in parts of the tumor. 


\section{References}

1 Coyne JD: Colonic carcinoma with granulomatous (sarcoid) reaction. J Clin Pathol 2002;55:708-709.

2 Grissom JR, Yamase HT, Prosser PR: Giant pheochromocytoma with sarcoidosis. Southern Med J 1979;72:1605-1607.

3 Menzin AW, You TT, Deger RB, Brooks JS, King SA: Sarcoidosis in a uterine leiomyoma. Intern J Gynecol Obstet 1995;48:79-84.

4 Prior C, Knight RA, Herold M, Ott G, Spiteri MA: Pulmonary sarcoidosis: patterns of cytokine release in vitro. Eur Respir J 1996;9:47-53.

5 Heffernan MP, Smoller BR, Kohler S: Cutaneous epithelioid angioleiomyoma. Am J Dermatopathol 1998;20:213-217.

6 Stavros A, Konstantina V, Elias K, Demetrios AS: IL-1 cytokines in cardiovascular disease: diagnostic, prognostic and therapeutic implications. Cardiovasc Hematol Agents Med Chem 2008;6:150-158.

7 Warner SJ, Libb P: Human vascular smooth muscle cells. Target for and source of tumor necrosis factor. J Immunol 1989;142:100-109. 